\title{
Myocardial injury and fibrogenesis: extracellular volume expansion is associated with elevated Galectin-3 levels in patients with myocarditis
}

\author{
Lukas Radziwolek*, Ulf K Radunski', Katharina Koopmann', Sebastian Bohnen', Tanja Zeller', Gunnar Lund², \\ Aljoscha D Krull', Nina Hauschild ${ }^{1}$, Christian Stehning ${ }^{3}$, Gerhard Adam ${ }^{1}$, Stefan Blankenberg ${ }^{1}$, Kai Muellerleile ${ }^{1}$
}

From 17th Annual SCMR Scientific Sessions

New Orleans, LA, USA. 16-19 January 2014

\section{Background}

Myocarditis subsumes a variety of entities, including diverse courses from complete healing to dilated cardiomyopathy with severe myocardial fibrosis. T1-mapping cardiovascular magnetic resonance (CMR) has the ability to quantify myocardial extracellular volume (ECV) as a surrogate of acute and chronic myocardial injury. Galectin-3 is an important mediator of fibrogenesis and contributes to adverse left ventricular (LV) remodeling. This study evaluated, if myocardial ECV expansion is linked to Galectin-3 levels in patients with myocarditis.

\section{Methods}

Galectin-3 blood levels were measured in 20 patients with myocarditis using a commercially available chemiluminescent microparticle immunoassay (ARCHITECT Galectin-3, Abbott Germany). T1 quantification was performed at 1.5 Tesla using the modified Look-Locker inversion-recovery (MOLLI) sequence before and 15 minutes after administration of $0.075 \mathrm{mmol} / \mathrm{kg}$ gadolinium-BOPTA. Global myocardial ECV was calculated from $\mathrm{T} 1$ maps generated by a dedicated plug-in written for the OsiriX software.

\section{Results}

Median Galectin-3 level was $17.4 \mathrm{ng} / \mathrm{mL}$ (interquartile range 13.2 to $20.5 \mathrm{ng} / \mathrm{mL}$ ) and median global myocardial ECV was $29 \%$ (interquartile range 26 to $33 \%$ ) in the study population. There was a significant correlation between Galectin-3 levels and global myocardial ECV $(\mathrm{r}=$ $0.50 ; \mathrm{p}<0.05)$. In contrast, no significant correlation was found between Galectin-3 levels and LV end-diastolic volumes $(\mathrm{r}=-0.08 ; \mathrm{p}=\mathrm{ns}), \mathrm{LV}$ end-systolic volumes $(\mathrm{r}=$ 0.06; $\mathrm{p}=\mathrm{ns}), \mathrm{LV}$ stroke volumes $(\mathrm{r}=-0.33 ; \mathrm{p}=\mathrm{ns}) ; \mathrm{LV}$ ejection fractions $(\mathrm{r}=-0.11 ; \mathrm{p}=\mathrm{ns})$, Troponin $\mathrm{T}$ levels $(\mathrm{r}=0.20 ; \mathrm{p}=\mathrm{ns})$ or NT-proBNP levels $(\mathrm{r}=0.28 ; \mathrm{p}=\mathrm{ns})$, respectively.

\section{Conclusions}

Myokardial ECV expansion, as a surrogate for myocardial injury, is associated with increased Galectin-3 levels, indicating activated fibrogenesis in patients with myocarditis. Combining Galectin-3 measurements with ECV-imaging could improve risk stratification beyond conventional imaging parameters or biomarkers in these patients.

\section{Funding}

Marija-Orlovic-Foundation.

\begin{abstract}
Authors' details
${ }^{1}$ Cardiology, University Heart Center, Hamburg, Germany. ${ }^{2}$ Radiology, University Medical Center, Hamburg, Germany. ${ }^{3}$ Philips Research, Hamburg, Germany.
\end{abstract}

Published: 16 January 2014

doi:10.1186/1532-429X-16-S1-P290

Cite this article as: Radziwolek et al:: Myocardial injury and fibrogenesis:

extracellular volume expansion is associated with elevated Galectin-3

levels in patients with myocarditis. Journal of Cardiovascular Magnetic

Resonance 2014 16(Suppl 1):P290.

${ }^{1}$ Cardiology, University Heart Center, Hamburg, Germany

Full list of author information is available at the end of the article 